\title{
Uso do exame radiográfico no diagnóstico de Osteopatia Hipertrófica Pulmonar (OHP): relato de três casos em cães
}

\section{Use of radiographic examinations in the diagnosis of Pulmonary Hypertrophic Osteopathy (OHP): report on three cases in dogs}

\section{Resumo}

A Osteopatia Hipertrófica Pulmonar (OHP) é uma patologia caracterizada por uma neoformação óssea decorrente da resposta periosteal nos ossos longos e nas extremidades, secundária a doenças intratorácicas como tumores primários ou metastáticos; doenças pielogranulomatosas e neoplasias primárias em órgãos pélvicos. Pode afetar várias espécies e não possui predisposição racial e nem relação com o biótipo; porém, é comumente encontrada em animais idosos. Os sinais clínicos comuns são: aumento da temperatura e edema nos membros acometidos, relutância ao movimento e claudicação; contudo, alguns sinais podem estar ligados à doença primária. A fisiopatologia ainda não está totalmente esclarecida; porém, existem diferentes teorias para explicar a ocorrência. O presente trabalho relata três casos, observados em cães atendidos, e diagnosticados como Osteopatia Hipertrófica Pulmonar em centros de Diagnóstico por Imagem.

\section{Summary}

Pulmonary Hypertrophic Osteopathy (OHP) is a condition characterized by a bone neoformation resulting from periosteal response in long bones and extremities. It is secondary to intrathoracic diseases such as primary or metastatic tumors; pyelo-granulomatous diseases and primary malignancies in the pelvic organs. It may affect several species, and has no racial predisposition or biotype relation, although it's commonly found in elder animals. The usual clinical signs are: increased temperature and edema in the affected limbs, reluctance to move and limping. However, some signs can be related to the primary disease. The pathophysiology is not yet fully understood, but there are different theories that explain the disease. This paper reports three cases seen in dogs and diagnosed as Pulmonary Hypertrophic Osteopathy in Diagnostic Imaging centers. 
Cíntia Zamponi Costa Candeias ${ }^{1}$

Débora Prado da Silva ${ }^{1}$

Ricardo Fuso Camargo ${ }^{2}$

Jefferson Douglas Soares Alves ${ }^{3}$

\section{Palavras-chave}

Cães. Metástases. Osso.

Periosteal. Osteopatia.

\section{Keywords}

Dogs. Metastasis. Bone.

Periosteal. Osteopathy.

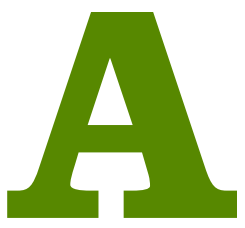

Osteopatia Hipertrófica Pulmonar (OHP) é uma proliferação desordenada e generalizada do periósteo que acomete com maior frequência os ossos longos e suas extremidades (BERRY; LOVE; THRALL, 2002), caracterizada como uma neoformação óssea ao redor das diáfises, metatarsos e metacarpos (BURK; ACKERMAN, 1996; FOSSUM, 2008; HERMETO et al., 2013).

A OHP pode ser observada tanto na medicina humana quanto na veterinária, com descrições relatadas em cavalos, vacas, ovelhas, gatos, aves domésticas e, principalmente, em cães (FILGUEIRAS et al., 2002; HEADLEY et al., 2005; TROST et al., 2012).

As principais causas da OHP estão relacionadas às neoplasias torácicas metastáticas e, menos frequentemente, a tumores primários; o que permite a sua definição como síndrome paraneoplásica (FILGUEIRAS et al., 2002; FOSSUM, 2008; PICCIONELLO et al., 2012). Algumas doenças pielogranulomatosas, como nocardiose e tuberculose; tumores em órgãos pélvicos, como na vesícula urinária e ovários, sem apresentarem metástase pulmonar; abscessos abdominais, dirofilariose e espirocercose já foram relatados como causas da OHP ou de predisposição para a mesma (BERRY; LOVE; THRALL, 2002; HEADLEY et al., 2005; HERMETO et al., 2013).

Grillo et al. (2007) e Borin et al. (2011) destacaram que a fisiopatologia da OHP ainda não foi totalmente esclarecida. Contudo, 
algumas teorias procuram elucidar a origem da doença. A teoria mais consistente seria o aumento da irrigação vascular periférica dos ossos longos, secundariamente à vasodilatação mediada pela estimulação neurovascular do nervo vago e dos intercostais, como resposta às alterações pulmonares existentes (FILGUEIRAS et al., 2002; FOSSUM, 2008). Assim, o periósteo reage, formando calcificações nas corticais dos ossos longos. Entretanto, existem outras teorias que citam ação hormonal, hipóxia, fatores humorais ou a sua combinação (MENEZES et al., 2012).

Cães de qualquer raça ou tamanho podem ser afetados, mas, como a OHP está normalmente associada a neoplasias, ela é comumente encontrada em animais idosos. A sua ocorrência em gatos é rara (HEADLEY et al., 2005; FOSSUM, 2008).

Os sinais clínicos mais comuns da OHP são: edema e temperatura elevada nas extremidades dos membros acometidos, relutância aos movimentos, sensibilidade dolorosa exacerbada e, consequentemente, claudicação (KEALY; McALLISTER, 2005; MENEZES et al., 2012; HERMETO et al., 2013). Alguns sinais clínicos podem estar ligados à doença primária, como: anorexia, tosse, hipertermia, dispneia ou taquipneia.

Dentre os sinais radiográficos mais encontrados na OHP são referidos: neoformação periosteal bilateral e simétrica nos ossos longos e extremidades, sendo os ossos do carpo e do tarso menos afetados, sem o comprometimento das articulações (OWENS; BIERY, 1998; FILGUEIRAS et al., 2002; MENEZES et al., 2012); aumento de volume das partes moles e deposição óssea mais frequente, de aspecto irregular, denominada "paliçada” (KEALY, 1987; KEALY; McALLISTER, 2005).

O tratamento da OHP consiste na remoção da causa primária, que resulta na regressão dos sinais clínicos em alguns animais, e também parcial ou totalmente das neoformações periosteais. Outra opção é a transecção do nervo vago, que também pode provocar a regressão da doença (FILGUEIRAS et al., 2002; HERMETO et al., 2013).

\section{Relato de Casos}

Foram encaminhados dois pacientes ao setor de Diagnóstico por Imagem do Hospital Veterinário "Vicente Borelli", Centro Universitário da Fundação de Ensino Octávio Bastos (UNIFEOB - São João da Boa Vista), para a realização de um exame radiográfico da cavidade torácica, para avaliação da silhueta cardíaca e campos pulmonares. Um terceiro paciente foi atendido no Centro de Diagnóstico por Imagem - CEDIVET, localizado na cidade de Rio Claro - SP.

\section{CASOS}

Animal 1: Fêmea, canina, raça Poodle, de 11 anos de idade. Com a realização do exame radiográfico da cavidade torácica, nas projeções lateral direita (LL) e ventrodorsal (VD), foi observado aumento global da silhueta cardíaca e presença de inúmeras estruturas arredondadas de densidade homogênea em região de lobos pulmonares médios e caudais, das quais a maior medindo $4,8 \times 4,8 \mathrm{~cm}$ de diâmetro, caracterizando um padrão intersticial nodular, imagem compatível com metástases pulmonares (Figuras 1 e 2).
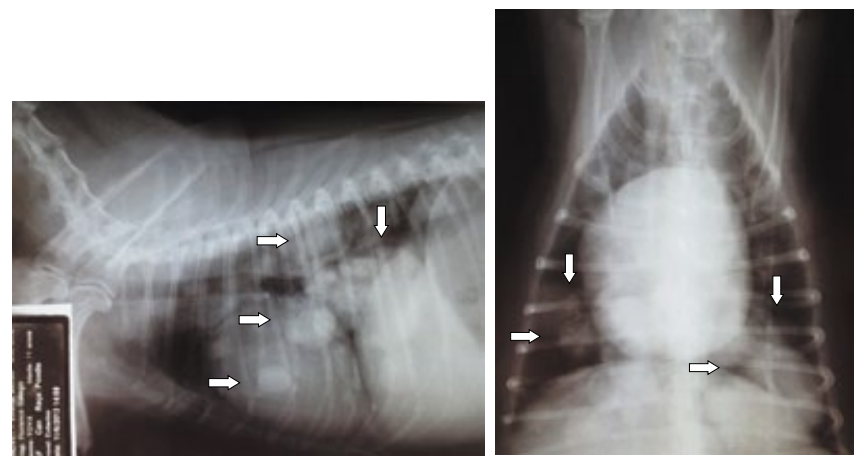

Figuras 1 e 2 - Radiografias torácicas (LL e VD), mostrando aumento global da silhueta cardíaca e estruturas arredondadas de densidade homogênea (nódulos) em lobos médios e caudais, indicando metástase pulmonar (setas brancas)

Fonte: Arquivo pessoal

Devido ao aumento de volume e sensibilidade dolorosa nos membros torácicos e pélvicos, relatado pela proprietária, optou-se pela realização do exame radiográfico desses membros nas projeções médio-lateral e craniocaudal. Foi constatada, então, a reação periosteal regular em úmeros, rádios, ulnas, metacarpos e falanges dos membros torácicos (Figura 3) e em tíbias, fíbulas, metatarsos e falanges dos membros pélvicos (Figura 4), com edema de partes moles em todos os membros estudados, o qual não era visualizado devido à quantidade de pêlo do animal.
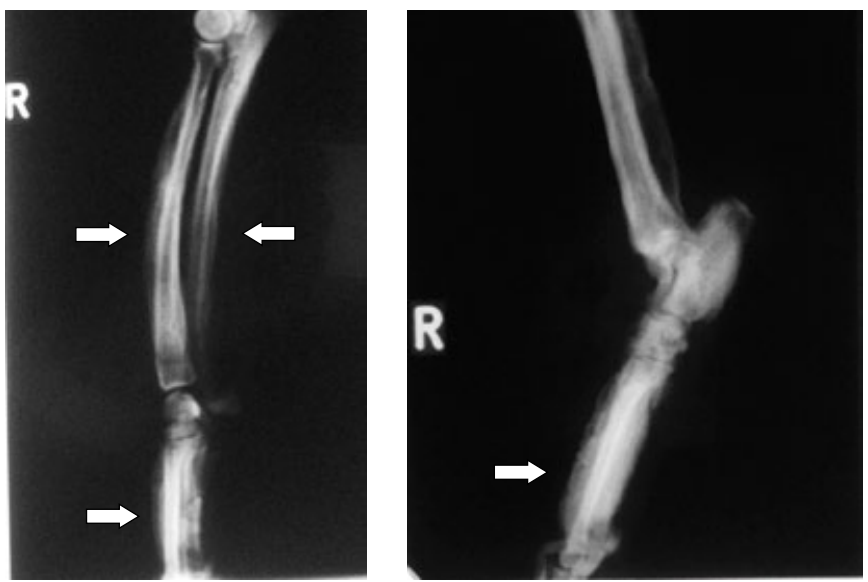

Figuras 3 e 4 - Radiografia dos membros torácico e pélvico (LL) com presença de reação periosteal em margem cranial do rádio e caudal da ulna (setas brancas) e margem dorsal dos metacarpos do membro torácico direito. Reação periosteal em margem dorsal dos metatarsos do membro pélvico direito

Fonte: Arquivo Pessoal 
De acordo com os achados radiográficos das regiões estudadas, juntamente com os achados clínicos, o caso foi diagnosticado como Osteopatia Hipertrófica Pulmonar. Uma vez que o paciente foi encaminhado apenas para o exame de imagem, a conduta terapêutica ficou restrita ao médico veterinário responsável pelo caso. Segundo a proprietária, o animal veio a óbito após 50 dias.

Animal 2: Macho, canino, raça Labrador, 17 anos de idade. Os exames radiográficos da cavidade torácica, nas projeções lateral direita (LL) e ventrodorsal (VD), revelaram a presença de uma estrutura de densidade homogênea com aproximadamente $18,0 \mathrm{~cm}$ x 15,0 cm de diâmetro em hemitórax direito, deslocando a silhueta cardíaca para a esquerda, considerada como uma massa intratorácica (Figuras 5 e 6).
Analisando-se a radiografia na projeção lateral direita (Figuras 5 e 6), observou-se a presença de reação periosteal na diáfise do úmero. Consequentemente, sugeriu-se a realização de radiografias dos membros torácicos e pélvicos para melhor elucidação e conclusão do caso.

Nas projeções lateral direita, lateral esquerda e craniocaudal dos membros torácicos e pélvicos, foram observadas acentuadas reações periosteais por toda a extensão dos ossos longos, e o diagnóstico do segundo caso foi firmado como Osteopatia Hipertrófica Pulmonar (Figuras 7, 8, 9 e 10).

Após duas semanas da realização do exame, o médico veterinário responsável informou ao setor de Imagem o óbito do animal.

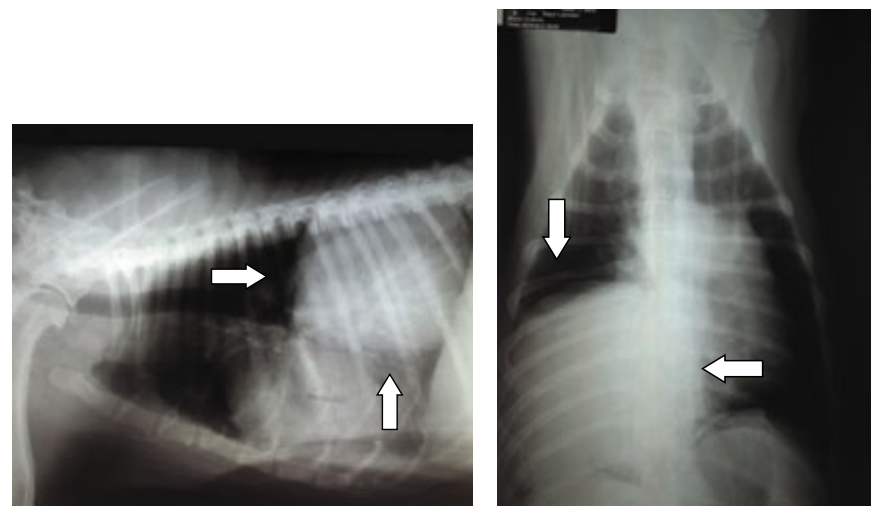

Figuras 5 e 6 - Radiografias Torácicas (LL e VD) evidenciando uma estrutura de densidade homogênea $(18,0 \times 15,0 \mathrm{~cm})$ em hemitórax direito (setas brancas), dificultando a visualização da silhueta cardíaca e da crura diafragmática direita Fonte: Arquivo Pessoal

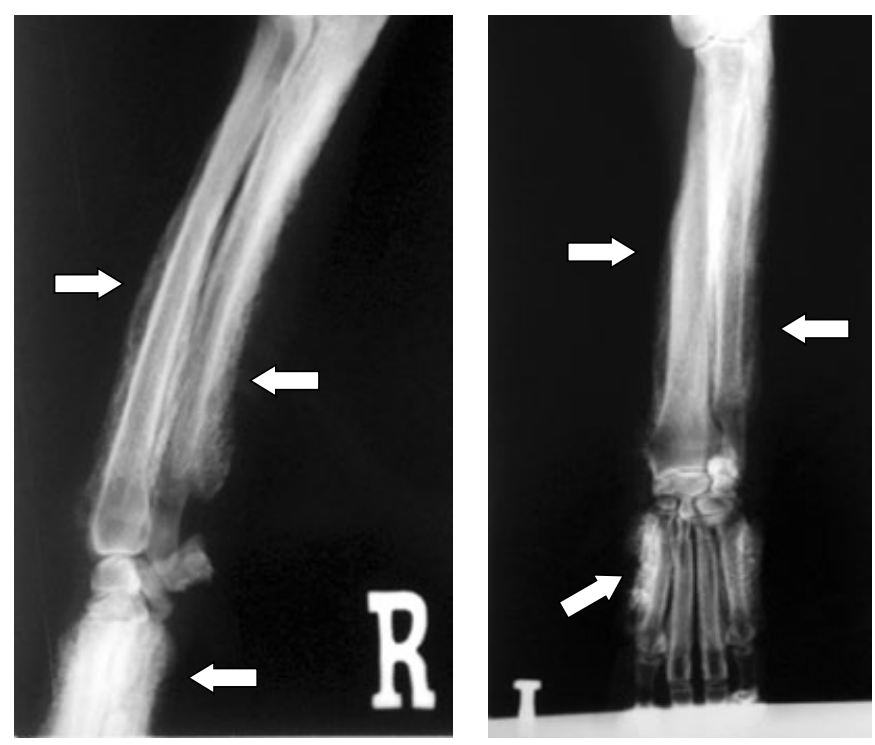

Figuras 7 e 8 - Radiografia dos membros torácicos nas projeções lateral direita e craniocaudal - Observam-se acentuadas reações periosteais regulares em rádios, ulnas e metacarpos dos membros supracitados (setas brancas) Fonte: Arquivo Pessoal
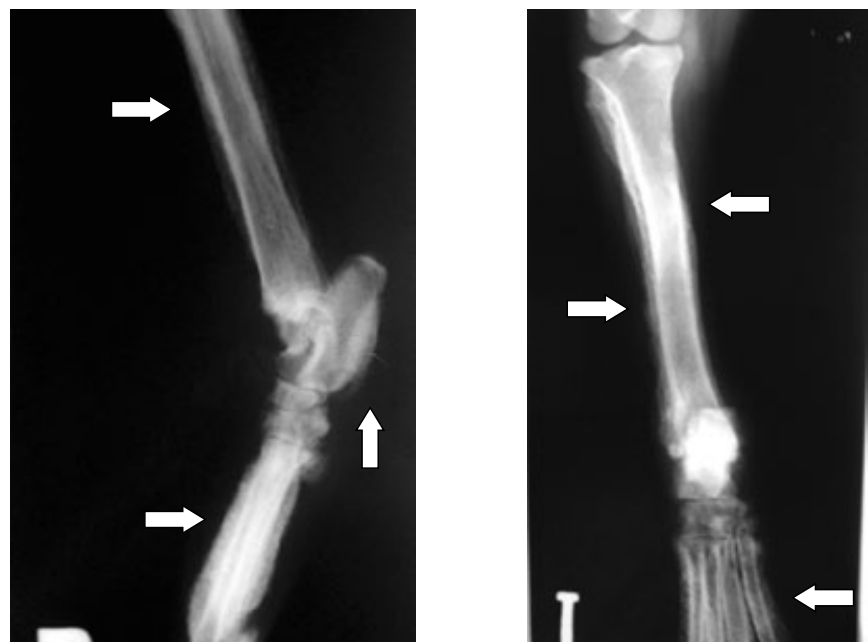

Figuras 9 e 10 - Radiografias dos membros pélvicos nas projeções lateral direita e craniocaudal - Observam-se reações periosteais regulares em tíbias, calcâneo e metatarsos (setas brancas) dos membros supracitados Fonte: Arquivo Pessoal

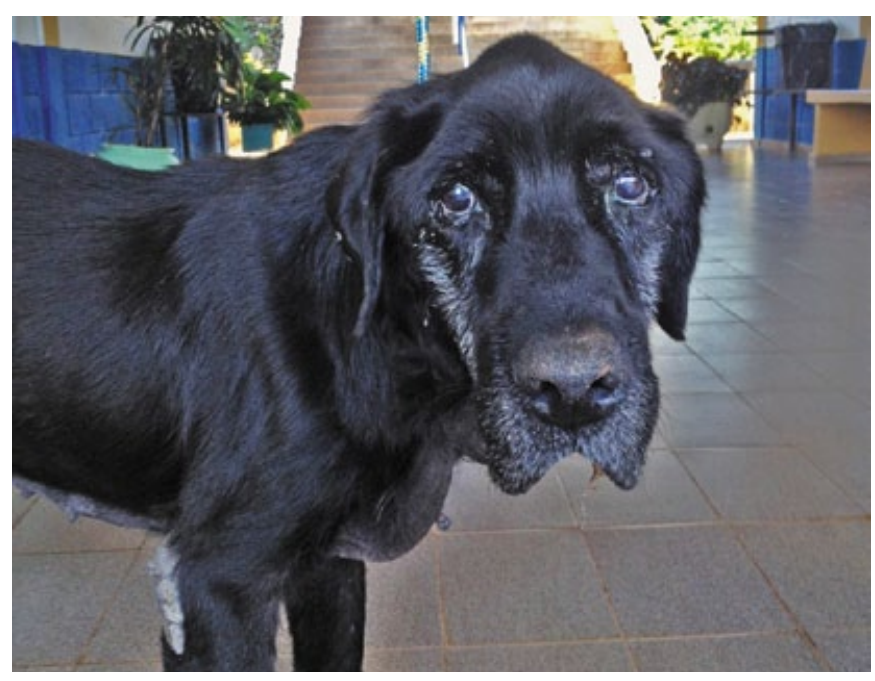

Figura 11 - Animal atendido no setor de imagens da Unifeob Fonte: Arquivo Pessoal 
Animal 3: Fêmea, canina, raça Cocker, 13 anos de idade. Animal atendido no centro de diagnóstico por imagem Cedivet, onde foram realizadas radiografias computadorizadas da cavidade torácica e dos membros pélvicos. Foram observados: aumento de volume de partes moles e reações periosteais em ossos longos e suas extremidades, e discretos nódulos em campos pulmonares; o que permitiu o estabelecimento do diagnóstico de OHP (Figuras 12 e 13).
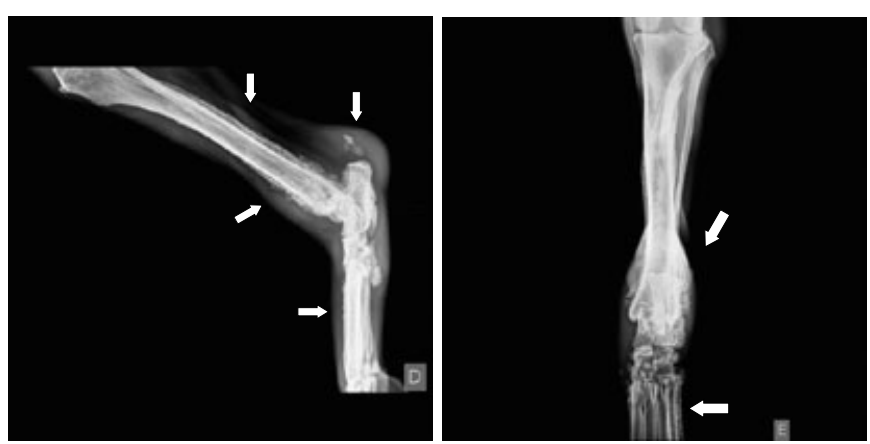

Figuras 12 e 13 - Radiografias das tíbias e metatarsos (LL e Craniocaudal) mostrando aumento de volume de partes moles e acentuada reação periosteal irregular nas extremidades dos ossos analisados (setas brancas)

Fonte: Arquivo Pessoal

\section{Discussão e Conclusão}

Os animais em estudo apresentaram neoformação periosteal nas diáfises e falanges dos ossos longos das extremidades; o que ocasionou sensibilidade dolorosa nos membros acometidos e dificuldade de locomoção, condição também relatada por Filgueiras et al. (2002); Trost et al. (2012) e Hermeto et al. (2013).

A OHP tem como causa principal as neoplasias intratorácicas primárias ou metastáticas; porém, outras patologias como doenças pielogranulomatosas (tuberculose); neoplasias em órgãos pélvicos e abscessos abdominais podem determinar o seu desenvolvimento (FILGUEIRAS et al., 2002; HEADLEY et al., 2005; MENEZES et al., 2012); o que foi demonstrado e confirmado pelas imagens radiográficas dos animais estudados.

$\mathrm{O}$ exame radiográfico efetuado revelou a neoformação óssea periosteal de aspecto liso ou irregular (paliçada) distribuída simetricamente nas diáfises e falanges dos ossos longos. Geralmente os metacarpos e metatarsos são os primeiros e mais afetados; já os carpos, tarsos e articulações são menos atingidos. Os aspectos radiográficos observados concordam com os referidos por Kevin (1987); Kealy e Mcallister (2005) e Borin et al. (2011), que relataram que estes tendem a regredir ou mesmo sumir, quando a causa principal é retirada ou tratada com sucesso. Porém, nos três casos relatados não houve o acompanhamento clínico após o exame de imagem.
Filgueiras et al. (2002) destaca que o diagnóstico definitivo da OHP deve assentar-se na correlação entre sinais clínicos e os achados radiográficos, e que o prognóstico dependerá fundamentalmente do tipo de lesão existente, bem como da sua extensão e evolução. 


\section{Referências}

1. BERRY, C. R.; LOVE, N. E.; THRALL, D. E. Appendicular skeleton-canine and feline In: THRALL, D. E. Textbook of veterinary diagnostic radiology. 4. ed. Philadelphia, Saunders, 2002. $758 \mathrm{p}$

2. BORIN, S.; ZUCCOLOTTO CRIVELENTI, L.; GÓMEZ ORTIZ, E. M.; FERREIRA, F. A. Osteopatia hipertrófica canina: relato de caso. Revista de Investigaciones Veterinarias del Perú, v. 22, n. 2, p. 155, 2011

3. BURK, R. L.; ACKERMAN, N. The appendicular skeleton. In: Small animal radiology and ultrasonography. 2. ed. Philadelphia: Saunders, 1996. p. 488-491.

4. FILGUEIRAS, R. R.; SILVA, J. C. P.; VILORIA, M. I. V.; ODENTHAL, M. E.; DUARTE, T. S.; LAVOR, M. S. L. Osteopatia hipertrófica em cão: relato de caso. Clínica Veterinária v. 36, p. $28-32,2002$.

5. FOSSUM, T. W. Outras doenças dos ossos e articulações. In: Cirurgia de pequenos animais. 3. ed. Rio de Janeiro: Elsevier, 2008. p. 1333-1334.

6. GRILLO, T. P.; BRANDÃO, C. V.; MAMPRIM, M. J.; JESUS, C. M. N.; SANTOS, T. C. MINTO, B. W. Hypertrophic osteopathy associated with renal pelvis transitional cell carcinoma in a dog. The Canadian Veterinary Journal, v. 48, n. 7, p. 745-747, 2007.

7. HEADLEY, S. A.; RIBEIRO, E. A.; SANTOS, G. J. V. G.; BETTINI, C. M.; MATTOS JUNIOR, E. Osteopatia hipertrófica canina associada com lesões extratorácicas. Ciência Rural, v. 35, n. 4, p. 941-944, 2005.

8. HERMETO, L. C.; FERNANDES, S. S. P.; JARDIM, P. H. A.; MATTEI, D. R.; PELISARI, T. Osteopatia hipertrófica pulmonar: alterações clínicas e radiográficas em um paciente canino. Archives of Veterinary Science, v. 18, n. 2, p. 50-55, 2013.

9. KEALY, J. K.; MCALLISTER, H. Ossos e articulações. In: Radiologia e ultrasonografia do cão e do gato. 3. ed. Barueri: Manole, 2005. p. 296.

10. KEVIN, K. J. Bones and Joints. In:___ Diagnostic radiology of the dog and cat. 2 ed. Philadelphia: Saunders, 1987. p. 418-420.

11. MENEZES, M. S.; LIMEIRA, S. V.; SUARES, D. V.; AMADO, L. V.; PEREIRA, J. J.; VEIGA, C. C. P. Avaliação ultrassonográfica pulmonar em cão com osteoartropatia hipertrófica: relato de caso. Veterinária e Zootecnia, v. 19, p. 68-70, 2012.

12. OWENS, J. M.; BIERY, D. N. Extremities In: ___. Radiographic interpretation for the small animal clinician. 2. ed. Baltimore: Williams \& Wilkins, 1998. p. 41-42.

13. PICCIONELLO, A. P.; DINI, F.; TAMBELLA, A. M.; CERQUeTELLA, M.; VULLO, C. Hypertrophic osteopathy associated with a bronchial foreign body (grass awn) in a dog: a case report. Veterinary Medicine, v. 57, n. 11, p. 618-621, 2012.

14.TROST, M. E.; KOMMERS, G. D.; SILVA, T. M.; IRIGOYEN, L. F.; FIGHERA, R. A. BARROS, C. S. L.; INKELMANN, M. A. Osteopatia hipertrófica em sete cães. Pesquisa Veterinária Brasileira, v. 32, n. 5, p. 424-429, 2012 